\title{
On the applicability of Lions' energy estimates in the analysis of discrete optimized Schwarz methods with cross points
}

\author{
Martin J. Gander ${ }^{1}$ and Felix Kwok ${ }^{2}$
}

\section{Introduction}

For a bounded open subset $\Omega \subset \mathbb{R}^{2}$, suppose we want to solve

$$
(\eta-\Delta) u=f \quad \text { on } \Omega, \quad u=g \quad \text { on } \partial \Omega
$$

for $\eta \geq 0$ using the optimized Schwarz method (OSM)

$$
\begin{aligned}
& (\eta-\Delta) u_{i}^{k}=\left.f\right|_{\Omega_{i}} \quad \text { on } \Omega_{i}, \quad u_{i}^{k}=\left.g\right|_{\partial \Omega_{i}} \quad \text { on } \partial \Omega_{i} \cap \partial \Omega \\
& \frac{\partial u_{i}^{k}}{\partial n_{i}}+p_{i j} u_{i}^{k}=\frac{\partial u_{j}^{k-1}}{\partial n_{i}}+p_{i j} u_{j}^{k-1} \quad \text { on } \Gamma_{i j} \text { for all } \Gamma_{i j} \neq \emptyset
\end{aligned}
$$

for $k=1,2, \ldots$ and $i=1, \ldots, n$, where $\Omega_{i} \subset \Omega$ are non-overlapping subdomains, $\Gamma_{i j}=\partial \Omega_{i} \cap \overline{\Omega_{j}}$ is the interface between $\Omega_{i}$ and an adjacent subdomain $\Omega_{j}, j \neq i$, and $p_{i j}>0$ are Robin parameters along $\Gamma_{i j}$. In [7], the powerful technique of energy estimates is used to show convergence of (2) for $\eta=0$ under very general settings. Similar techniques have been used to prove convergence results for other types of equations, cf. [2] for the Helmholtz equation and [5] for the time-dependent wave equation. While one often assumes that the proof carries over trivially to finiteelement discretizations, it has been reported in the literature (cf. $[9,8])$ that discrete OSMs can diverge when the domain decomposition contains cross points, i.e., when more than two subdomains share a common point. This is in apparent contradiction with Lions' proof, and such difficulties contribute to the limited use of OSMs in practice. The goal of this paper is to explain why the presence of cross points makes it possible for the discrete OSM to diverge despite the proof of convergence at the continuous level, and why this difference in behavior is generally unavoidable.

The remainder of the paper proceeds as follows. In Section 2, we recall Lions' energy estimate argument. In Section 3, we explain why it is impossible to convert the

Section de Mathématiques, Université de Genève \{Martin. Gander|Felix.Kwok\}@unige.ch 
continuous energy estimate into a discrete one in a generic way, without sacrificing continuity of the solutions across subdomain boundaries. In section 4 , we mention two modifications that preserve continuity of the discrete solutions, but both must be used with Krylov methods to avoid divergent iterations. Finally, we show in Section 5 that a Lions-type discrete estimate can only hold under very stringent conditions; thus, continuous estimates generally do not predict the behavior of discrete OSMs.

\section{Continous Energy Estimates}

We briefly recall the argument in [7] proving the convergence of (2). We assume $p_{i j}=p_{j i}$ to be a positive function that is bounded away from zero and defined on $\Gamma_{i j}=\Gamma_{j i}$. To show that (2) converges for all initial guesses, we first write the error equations

$$
\begin{aligned}
(\eta-\Delta) e_{i}^{k} & =0 \quad \text { on } \Omega_{i}, \quad e_{i}^{k}=0 \quad \text { on } \partial \Omega \cap \partial \Omega_{i}, \\
\frac{\partial e_{i}^{k}}{\partial n_{i}}+p_{i j} e_{i}^{k} & =\frac{\partial e_{j}^{k-1}}{\partial n_{i}}+p_{i j} e_{j}^{k-1} \quad \text { on } \Gamma_{i j} \text { for all } \Gamma_{i j} \neq \emptyset,
\end{aligned}
$$

where $e_{i}=u_{i}^{k}-\left.u\right|_{\Omega_{i}}$ with $u$ being the exact solution to (1). We then multiply the first equation in (3) by $e_{i}^{k}$ and integrate to get

$$
0=a_{i}\left(e_{i}^{k}, e_{i}^{k}\right)-\int_{\partial \Omega_{i}} e_{i}^{k} \frac{\partial e_{i}^{k}}{\partial n_{i}}=a_{i}\left(e_{i}^{k}, e_{i}^{k}\right)-\sum_{(i, j) \in E} \int_{\Gamma_{i j}} e_{i}^{k} \frac{\partial e_{i}^{k}}{\partial n_{i}},
$$

where the last sum is over all pairs of subdomains $(i, j)$ that share an interface, and $a_{i}\left(u_{i}, v_{i}\right)=\int_{\Omega_{i}}(\nabla u \cdot \nabla v+\eta u v) d x$ is the energy bilinear form defined on subdomain $\Omega_{i}$, so that $a_{i}\left(e_{i}^{k}, e_{i}^{k}\right)=\int_{\Omega_{i}} \eta\left|e_{i}^{k}\right|^{2}+\left|\nabla e_{i}^{k}\right|^{2} d x \geq 0$ is the energy of the error on subdomain $\Omega_{i}$. We now rewrite the product term as

$$
e_{i}^{k} \frac{\partial e_{i}^{k}}{\partial n_{i}}=\frac{1}{4 p_{i j}}\left[\left(\frac{\partial e_{i}^{k}}{\partial n_{i}}+p_{i j} e_{i}^{k}\right)^{2}-\left(-\frac{\partial e_{i}^{k}}{\partial n_{i}}+p_{i j} e_{i}^{k}\right)^{2}\right]=:\left(T_{+i j}^{k}\right)^{2}-\left(T_{-i j}^{k}\right)^{2},
$$

where $T_{ \pm i j}^{k}=\frac{1}{\sqrt{4 p_{i j}}}\left( \pm \frac{\partial e_{i}^{k}}{\partial n_{i}}+p_{i j} e_{i}^{k}\right)$. Since $\frac{\partial e_{j}^{k}}{\partial n_{i}}=-\frac{\partial e_{j}^{k}}{\partial n_{j}}$ on $\Gamma_{i j}$, the interface condition in (3) can be written as $T_{+i j}^{k}=T_{-j i}^{k-1}$, which means

$$
a_{i}\left(e_{i}^{k}, e_{i}^{k}\right)=\sum_{(i, j) \in E} \int_{\Gamma_{i j}}\left[\left(T_{+i j}^{k}\right)^{2}-\left(T_{-i j}^{k}\right)^{2}\right] d s=\sum_{(i, j) \in E} \int_{\Gamma_{i j}}\left[\left(T_{-j i}^{k-1}\right)^{2}-\left(T_{-i j}^{k}\right)^{2}\right] d s .
$$

Thus,

$$
a_{i}\left(e_{i}^{k}, e_{i}^{k}\right)+\sum_{(i, j) \in E} \int_{\Gamma_{i j}}\left(T_{-i j}^{k}\right)^{2} d s=\sum_{(i, j) \in E} \int_{\Gamma_{i j}}\left(T_{-j i}^{k-1}\right)^{2} d s .
$$

If we sum (4) through all subdomains $i$, we get

$$
\sum_{i=1}^{N} a_{i}\left(e_{i}^{k}, e_{i}^{k}\right)+\sum_{i=1}^{N} \sum_{(i, j) \in E} \int_{\Gamma_{i j}}\left(T_{-i j}^{k}\right)^{2} d s=\sum_{i=1}^{N} \sum_{(i, j) \in E} \int_{\Gamma_{i j}}\left(T_{-j i}^{k-1}\right)^{2} d s .
$$


We can now sum (5) over $k$ and simplify to get

$$
\sum_{k=0}^{K} \sum_{i=1}^{N} a_{i}\left(e_{i}^{k}, e_{i}^{k}\right)+B^{K}=B^{0}
$$

where $B^{k}:=\sum_{i=1}^{N} \sum_{(i, j) \in E} \int_{\Gamma_{i j}}\left(T_{-i j}^{k}\right)^{2} d s \geq 0$. Since $B^{K} \geq 0$ and each $a_{i}\left(e_{i}^{k}, e_{i}^{k}\right) \geq 0$, we see that $\sum_{k=0}^{K} a_{i}\left(e_{i}^{k}, e_{i}^{k}\right) \leq B^{0}$ for all $i$ and all $K$; hence $a_{i}\left(e_{i}^{k}, e_{i}^{k}\right) \rightarrow 0$ as $k \rightarrow \infty$ for all $i$. This implies that $\left\|e_{i}^{k}\right\|_{H^{1}\left(\Omega_{i}\right)} \rightarrow 0$ when $\eta>0$, so $\left.u_{i} \rightarrow u\right|_{\Omega_{i}}$ in the $H^{1}$ norm. A similar argument holds for $\eta=0$. Note that the possible presence of cross points does not cause any difficulty in the proof, since they form a subset of measure zero in $\partial \Omega_{i}$ and thus do not contribute to the boundary terms when integrating by parts.

\section{Finite Element Discretization}

We now try to mimic Lions' proof in the finite element case. The finite element method uses the weak form of (2), i.e., we must multiply the PDE by a test function $\phi$ and integrate by parts. The problem becomes

$$
\begin{aligned}
& \text { Find } u_{i} \in V^{h} \subset H^{1}\left(\Omega_{i}\right) \text { s.t. for all } \phi \in W^{h} \subset H_{0}^{1}(\Omega) \cap H^{1}\left(\Omega_{i}\right), \\
& \qquad \int_{\Omega_{i}}\left(\nabla \phi \cdot \nabla u_{i}^{k}+\eta \phi u_{i}^{k}\right)-\int_{\partial \Omega_{i}} \phi \frac{\partial u_{i}^{k}}{\partial n_{i}}=\int_{\Omega_{i}} \phi f .
\end{aligned}
$$

We now suppose that $\phi$ is a basis function corresponding to a degree of freedom along $\Gamma_{i j}$, whose support does not contain any cross points, see Figure 1(a). To obtain an expression for $\int_{\partial \Omega_{i}} \phi \frac{\partial u_{i}^{k}}{\partial n_{i}}$, we multiply the interface condition by $\phi$ and integrate to get

$$
\int_{\Gamma_{i j}} \phi\left(\frac{\partial u_{i}^{k}}{\partial n_{i}}+p u_{i}^{k}\right)=\int_{\Gamma_{i j}} \phi\left(\frac{\partial u_{j}^{k-1}}{\partial n_{i}}+p u_{j}^{k-1}\right) .
$$

Substituting into (7) gives

$$
a_{i}\left(\phi, u_{i}^{k}\right)+\int_{\Gamma_{i j}} \phi p u_{i}^{k}-\int_{\Gamma_{i j}} \phi \frac{\partial u_{j}^{k-1}}{\partial n_{i}}=\int_{\Omega_{i}} \phi f+\int_{\Gamma_{i j}} \phi p u_{j}^{k-1} .
$$

Thus, we are faced with the same problem of finding an expression of $\int_{\Gamma_{i j}} \phi \frac{\partial u_{j}^{k-1}}{\partial n_{i}}$. Fortunately, we can use the weak form of the PDE from $\Omega_{j}$

$$
a_{j}\left(\phi, u_{j}^{k-1}\right)-\int_{\partial \Omega_{j}} \phi \frac{\partial u_{j}^{k-1}}{\partial n_{j}}=\int_{\Omega_{j}} \phi f .
$$

Since $n_{i}=-n_{j}$ on $\Gamma_{i j}$, adding (9) and (10) and rearranging gives

$$
a_{i}\left(\phi, u_{i}^{k}\right)+\int_{\Gamma_{i j}} \phi p u_{i}^{k}=\int_{\Omega_{i}} \phi f-a_{j}\left(\phi, u_{j}^{k-1}\right)+\int_{\Gamma_{i j}} \phi p u_{j}^{k-1},
$$

which is just the usual block-Jacobi splitting of the stiffness matrix along $\Gamma_{i j}$. 


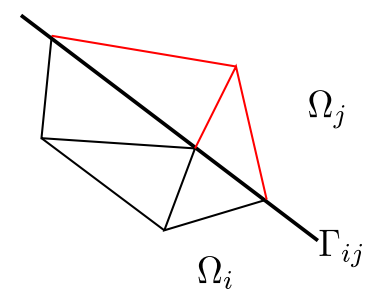

(a)

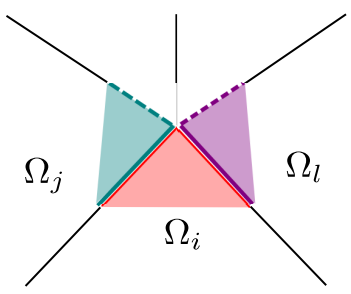

(b)

Fig. 1 Finite element discretization (a) without cross points and (b) with a cross point.

Now assume that the support of $\phi$ contains cross points, see Figure 1(b). Here $\Omega_{i}$ is adjacent to two distinct subdomains $\Omega_{j}$ and $\Omega_{l}, j \neq l$, and $\phi$ is non-zero on all three subdomains. Since the two parts of the interface, $\Gamma_{i j}$ and $\Gamma_{i l}$, must satisfy different interface conditions, we must separate $\int_{\partial \Omega_{i}} \phi \frac{\partial u_{i}^{k}}{\partial n}$ into contributions along $\Gamma_{i j}$ and $\Gamma_{i l}$,

$$
a_{i}\left(\phi, u_{i}^{k}\right)-\int_{\Gamma_{i j}} \phi \frac{\partial u_{i}^{k}}{\partial n_{i}}-\int_{\Gamma_{i l}} \phi \frac{\partial u_{i}^{k}}{\partial n_{i}}=\int_{\Omega_{i}} \phi f,
$$

where the two boundary terms can be replaced using the interface conditions

$$
\begin{aligned}
\int_{\Gamma_{i j}} \phi\left(\frac{\partial u_{i}^{k}}{\partial n_{i}}+p u_{i}^{k}\right) & =\int_{\Gamma_{i j}} \phi\left(\frac{\partial u_{j}^{k-1}}{\partial n_{i}}+p u_{j}^{k-1}\right), \\
\int_{\Gamma_{i l}} \phi\left(\frac{\partial u_{i}^{k}}{\partial n_{i}}+p u_{i}^{k}\right) & =\int_{\Gamma_{i l}} \phi\left(\frac{\partial u_{l}^{k-1}}{\partial n_{i}}+p u_{l}^{k-1}\right) .
\end{aligned}
$$

But now we cannot use the PDE on $\Omega_{j}$ and $\Omega_{l}$ to eliminate the terms $\int_{\Gamma_{i j}} \phi \frac{\partial u_{j}^{k-1}}{\partial n_{i}}$ and $\int_{\Gamma_{i l}} \phi \frac{\partial u_{l}^{k-1}}{\partial n_{j}}$, since the PDE on $\Omega_{j}$ will involve the Robin trace of $u_{j}$ with another subdomain that is not $\Omega_{i}$, and similarly for $\Omega_{l}$. Thus, it is impossible to discretize (2) without introducing extra unknowns to represent the combined Robin traces (integrated against a test function) for each subdomain at the cross point. Note that, unlike in the continuous setting, the discrete Robin traces are integrated along a subset of $\partial \Omega_{i}$ of non-zero measure straddling both interfaces $\Gamma_{i j}$ and $\Gamma_{i l}$, and piecewise interface quantities are not available. Thus, the traces cannot be transmitted separately along $\Gamma_{i j}$ and $\Gamma_{i l}$, unlike in the continuous case.

One way of circumventing the problem is to use mortar methods $[1,6]$, which are designed for non-conforming grids. In these methods, the interface conditions are imposed using mortar functions, which have one fewer degree of freedom at the ends of intervals. Thus, there is no equation at the cross point, and the problem of unavailable Robin traces goes away. However, since the interface conditions are only enforced weakly, the method does not generally converge to the exact solution of the global FEM problem, but rather to a discontinuous solution (Figure 2) that is $O\left(h^{p}\right)$-accurate, where $p$ is the order of the finite element method. 


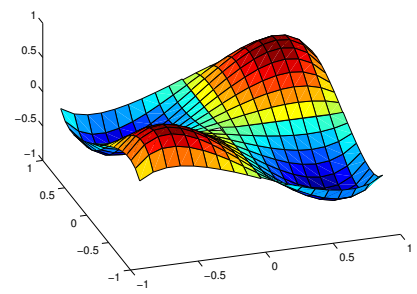

(a)

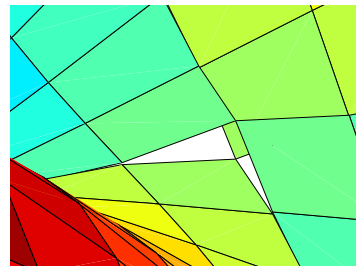

(b)

Fig. 2 (a) The solution of $-\Delta u=f$ with four subdomains on $\Omega=[-1,1]^{2}$, with right-hand side $f(x, y)=\sin (x y)$. The interface conditions are imposed using a mortar space. (b) Discontinuity of the composite solution near the origin.

\section{Two Lagrange Multiplier and Primal-Dual Methods}

If we want to formulate subdomain problems that are equivalent to the discrete global FEM problem, we need to introduce extra variables to represent the total Robin traces. Thus, at the cross point, we impose for each $\Omega_{i}$

$$
a_{i}\left(\phi, u_{i}^{k}\right)+\int_{\partial \Omega_{i}} p \phi \cdot u_{i}^{k}+\lambda_{i}^{k}=\int_{\Omega_{i}} \phi f,
$$

where $\lambda_{i}^{k}$ are Lagrange multipliers for ensuring consistency with the global problem. A cross point touching $r$ subdomains requires $r$ such Lagrange multipliers, so we also need $r$ constraints to be satisfied at convergence:

- Continuity constraints ( $r-1$ equations): at the cross point, we must have $u_{1}=$ $u_{2}=\cdots=u_{r}$.

- PDE constraint (1 equation): if we sum (12) over the $r$ subdomains and then subtract the global PDE $\sum_{i=1}^{r} a_{i}\left(\phi, u_{i}\right)=\int_{\Omega} \phi f$ from the result, we get

$$
\sum_{i=1}^{N} \int_{\partial \Omega_{i}} p \phi u_{i}+\sum_{i=1}^{N} \lambda_{i}=0 .
$$

This gives two types of algorithms:

1. Primal-Dual methods: the continuity constraints are enforced for every iteration. Thus, it suffices to introduce one extra variable (typically a coarse-grid basis function that has the value one at the cross point), and the PDE constraint is used as part of the coarse problem. This approach is similar to FETI-DP [3], except it is usually formulated with Neumann rather than Robin traces.

2. Two-Lagrange Multiplier methods: the $\lambda_{i}^{k}$ are retained, but the $u_{i}^{k}$ are eliminated using the PDE in the interior of the subdomain. This leads to a substructured problem formulated on the interface, which is then solved using a preconditioned Krylov method such as GMRES. This is known as the Two-Lagrange Multiplier (2LM) method and has been studied in detail in [8]. 


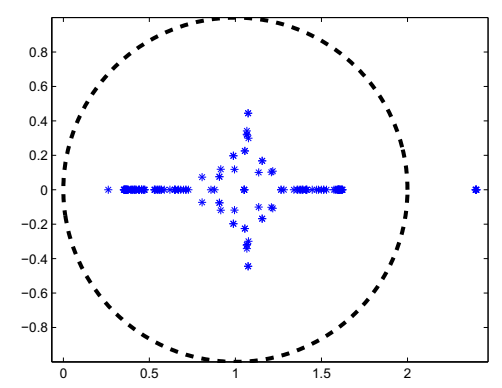

Fig. 3 Eigenvalues of the 2LM-preconditioned system for Poisson's equation $(\eta=0)$, using a $4 \times 4$ decomposition of the unit square with mesh size $h=1 / 64$ and Robin parameter $p=C / \sqrt{h}$ for all interface nodes.

Note that neither formulation is an exact discretization of (2) at cross points; thus, Lions' convergence analysis does not apply there. In fact, one can show [4] that the eigenvalues of the iteration matrix of the 2LM method may lie outside the unit disc when cross points are present, as seen in the $4 \times 4$ example shown in Figure 3. In such cases, the method would diverge. However, convergence can be restored if one uses Robin parameters with a different scaling at the cross points [4].

\section{Conditions for Existence of Discrete Energy Estimates}

To see what conditions are needed for Lions' estimates to hold in the discrete case, let us consider solving $-\Delta u=f$ on $\Omega=[-1,1]^{2}$ using $P^{1}$ finite elements on a structured triangular mesh. This yields the system $A u=f$, where $A$ is identical to the matrix obtained from finite differences. If we now divide $\Omega$ into four subdomains corresponding to the four quadrants of the plane, then an optimized Schwarz method must solve

$$
\left(A_{i}+L_{i}\right) u_{i}^{k}=g_{i}^{k} \quad \text { on each } \Omega_{i} .
$$

Here, $A_{i}$ is the partially assembled stiffness matrix for $\Omega_{i}, L_{i}$ corresponds to transmission conditions, and $g_{i}^{k}$ is a function of $f$ and $u_{j}^{k-1}$ for $j \neq i$. To define the discrete error function, let us write $u_{i}^{*}=\left.u^{*}\right|_{\Omega_{i}}$, where $u^{*}$ is the exact solution to $A u=f$. Then the error on $\Omega_{i}$ is $e_{i}^{k}=u_{i}^{k}-u_{i}^{*}$, with discrete energy $a_{i}\left(e_{i}^{k}, e_{i}^{k}\right)=\left(e_{i}^{k}\right)^{T} A_{i} e_{i}^{k}>0$ whenever $e_{i}^{k} \neq 0$, since each subdomain touches a Dirichlet boundary. Now observe that

$$
A_{i} e_{i}^{k}=A_{i} u_{i}^{k}-A_{i} u_{i}^{*}=A_{i} u_{i}^{k}-f_{i} \quad \text { at interior nodes. }
$$

Since the stencils of $A_{i}$ and $A$ coincide at interior nodes, we see that $A_{i} e_{i}^{k}$ must be zero away from the interfaces. Thus, we in fact have

$$
a_{i}\left(e_{i}^{k}, e_{i}^{k}\right)=\sum_{v \in \partial \Omega_{i} \backslash \partial \Omega} e_{i}^{k}(v) \cdot\left(A_{i} e_{i}^{k}\right)(v)=\sum_{v \in \partial \Omega_{i} \backslash \partial \Omega}\left[\left(T_{+i}^{k}(v)\right)^{2}-\left(T_{-i}^{k}(v)\right)^{2}\right],
$$


where $T_{ \pm i}^{k}(v)$ are the "Robin traces" at an interface point $v$ :

$$
T_{+i}^{k}(v)=\frac{1}{\sqrt{4 p}}\left[\left(A_{i} e_{i}^{k}\right)(v)+p e_{i}^{k}(v)\right], \quad T_{-i}^{k}(v)=\frac{1}{\sqrt{4 p}}\left[-\left(A_{i} e_{i}^{k}\right)(v)+p e_{i}^{k}(v)\right] .
$$

Hence, if we let $T_{+i}^{k}(v)=T_{-j}^{k-1}(v)$ at every point $v$ on the interface, then the energy estimate holds exactly the same way as in the continuous case, and we have convergence of the method. This allows us to deduce the correct interface conditions for $v$ away from the cross point. Using the definition $e_{i}^{k}=u_{i}^{k}-u_{i}^{*}$, we have

$$
\left(A_{i}\left(u_{i}^{k}-u_{i}^{*}\right)\right)(v)+p\left(u_{i}^{k}(v)-u_{i}^{*}(v)\right)=-\left(A_{j}\left(u_{j}^{k-1}-u_{j}^{*}\right)\right)(v)+p\left(u_{j}^{k-1}(v)-u_{j}^{*}(v)\right) .
$$

But since

$$
\left(A_{i} u_{i}^{*}\right)(v)+\left(A_{j} u_{j}^{*}\right)(v)=f(v)
$$

we can simplify (13) to get

$$
\left(A_{i} u_{i}^{k}\right)(v)+p u_{i}^{k}(v)=f(v)-\left(A_{j} u_{j}^{k-1}\right)(v)+p u_{j}^{k-1}(v) .
$$

In other words, we need

$$
\left(L_{i} u_{i}^{k}\right)(v)=p u_{i}^{k}(v), \quad g_{i}^{k}(v)=f(v)-\left(A_{j} u_{j}^{k-1}\right)(v)+p u_{j}^{k-1}(v) .
$$

On the other hand, if $v$ is a cross point, then (14) is no longer valid, since $f(v)$ is the sum of many subdomain contributions. Thus, it is in general impossible to find $L_{i}$ and $g_{i}^{k}$ such that the relation $T_{+i}^{k}(v)=T_{-j}^{k-1}(v)$ holds at the cross point for some $j$. In our model problem, however, the stencil at the cross point has a special form for the first and third quadrant:

$$
\begin{aligned}
& \left(A_{1} u_{1}^{*}\right)(0,0)=u^{*}(0,0)-\frac{1}{2} u^{*}(0, h)-\frac{1}{2} u^{*}(h, 0), \\
& \left(A_{3} u_{3}^{*}\right)(0,0)=u^{*}(0,0)-\frac{1}{2} u^{*}(0,-h)-\frac{1}{2} u^{*}(-h, 0) .
\end{aligned}
$$

Thus, we actually have $\left(A_{1} u_{1}^{*}\right)(0,0)+\left(A_{3} u_{3}^{*}\right)(0,0)=\frac{1}{2} f(0,0)$, a known quantity! A similar relation holds between $\Omega_{2}$ and $\Omega_{4}$, so it is actually possible to find transmission conditions at the cross point that satisfy the discrete energy estimate. For $\Omega_{1}$, this reads

$$
\left(A_{1} u_{1}^{k}\right)(v)+p u_{1}^{k}(v)=\frac{1}{2} f(v)-\left(A_{3} u_{3}^{k-1}\right)(v)+p u_{3}^{k-1}(v) .
$$

Figure 4 shows the convergence of the method for $p=\frac{\pi}{2 \sqrt{h}}$, which gives the optimal contraction factor $\rho=1-O(\sqrt{h})$, just as in the two-subdomain case. Since the discrete energy estimate holds, the converged subdomain solutions always coincide with the exact discrete solution $u^{*}$, unlike the mortar case. In general, discrete energy estimates can only be derived if for every cross point $v$, its set of neighbors can be partitioned into disjoint pairs $(i, j)$ such that $\left(A_{i} u_{i}^{*}\right)(v)+\left(A_{j} u_{j}^{*}\right)(v)=f_{i j}(v)$ can be calculated without knowing $u^{*}$. For cross points with wide stencils or an odd number of neighbors, this would not be possible. In such cases, the methods in Section 4 are still excellent choices in practice, but one cannot use Lions' estimates to deduce convergence for arbitrary positive Robin parameters $p$. 


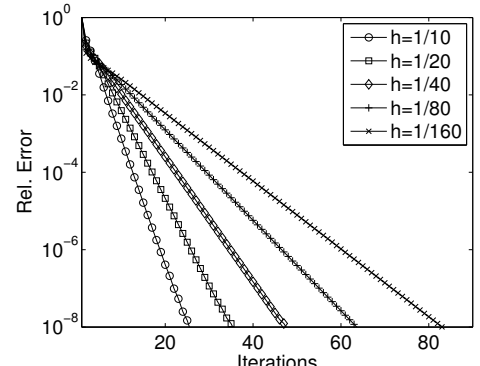

(a)

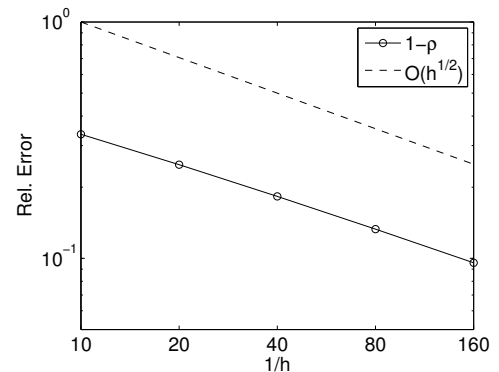

(b)

Fig. 4 (a) Convergence for different grid spacing $h$; (b) Contraction rate versus $h$.

Acknowledgment: the authors would like to thank Laurence Halpern for all the stimulating discussions concerning energy estimates.

\section{References}

[1] C. Bernardi, Y. Maday, and A. T. Patera. A new non conforming approach to domain decomposition: The mortar element method. In H. Brezis and J.-L. Lions, editors, Collège de France Seminar. Pitman, 1994.

[2] B. Després. Méthodes de décomposition de domaines pour les problèmes de propagation d'ondes en régime harmonique. $\mathrm{PhD}$ thesis, Univ. Paris IX Dauphine, 1991.

[3] C. Farhat, M. Lesionne, P. Le Tallec, K. Pierson, and D. Rixen. FETI-DP: a dual-primal unified FETI method - part I: a faster alternative to the two-level FETI method. Internat. J. Numer. Methods Engrg., 50:1523-1544, 2001.

[4] M. J. Gander and F. Kwok. Best Robin parameters for optimized Schwarz methods at cross points. submitted, 2011.

[5] M. J. Gander, L. Halpern, and F. Nataf. Optimized Schwarz waveform relaxation for the one dimensional wave equation. SIAM J. Numer. Anal., 41:16431681,2003

[6] M. J. Gander, C. Japhet, Y. Maday, and F. Nataf. A new cement to glue nonconforming grids with Robin interface conditions: The finite element case. In Domain Decomposition Methods in Science and Engineering, LNCSE 40, pages 259-266. Springer Verlag, 2004.

[7] P.-L. Lions. On the Schwarz alternating method III: a variant for nonoverlapping subdomains. In Third international symposium on domain decomposition methods for partial differential equations, pages 47-70, 1990.

[8] S. Loisel. Condition number estimates for the nonoverlapping optimized Schwarz method and the 2-Lagrange multiplier method for general domains and cross points. submitted, 2010.

[9] A. St-Cyr, D. Rosenberg, and S. D. Kim. Optimized Schwarz preconditioning for SEM based magnetohydrodynamics. In Domain Decomposition Methods in Science and Engineering XVIII, 2009. 